\title{
Effect of the Arch Support on the Strength of the Abductor Hallucis During the Toe Spread Out Exercise in Standing Position in Individuals With Pes Planus
}

\author{
In-cheol Jeon, PT, PhD, Ki-song Kim, PT, PhD \\ Department of Physical Therapy, College of Life and Health Science, Hoseo University, Asan, Korea
}

\author{
Article Info \\ Received June 21, 2020 \\ Revised July 18, 2020 \\ Accepted July 28, 2020 \\ Corresponding Author \\ Ki-song Kim \\ E-mail: kskim68@hoseo.edu \\ https://orcid.org/0000-0002-7184-3027
}

\section{Key Words}

Foot arch

Insole

Muscle activity

\begin{abstract}
Background: The longitudinal arch is important for individuals with pes planus. The toe spread out exercise (TSO) has been widely used to continuously support the longitudinal arch by increasing the abductor hallucis $(\mathrm{AbdH})$ muscle activation. However, the $\mathrm{AbdH}$ muscle is commonly lack of the sufficient activation during the TSO especially in individuals with pes planus.

Objects: This study was performed to investigate the effect of arch support on the muscle activity and strength of the AbdH during TSO in standing position in individuals with pes planus.

Methods: Twenty subjects with pes planus between 20 and 30 years of age participated in this study. The muscle activity and strength of the AbdH were measured using surface EMG system and the Smart KEMA tensiometer system. The AbdH muscle was evaluated during TSO between individuals with and without longitudinal arch support in standing position. The longitudinal arch was supported by using the insole. The paired t-test was used. The level of statistical significance was set at $\alpha=0.05$.
\end{abstract}

Results: The muscle activity and strength of the AbdH during TSO with arch support in standing position was significantly greater than that without arch support.

Conclusion: The muscle activity and strength of the AbdH during TSO in standing position can be influenced by the longitudinal arch support in individuals with pes planus. The $\mathrm{AbdH}$ strengthening during TSO in standing with arch support can be recommended especially in individuals with pes planus in the clinical settings.

\section{INTRODUCTION}

Pes planus is the impairment of foot as flattening of the longitudinal arch [1] and is misaligned foot condition as excessive pronation of the foot and abduction of the midfoot on the rear foot with valgus $[1,2]$. Pes planus is caused by overuse injuries, including patellofemoral pain syndrome [2], and plantar fasciitis [1]. The consideration of normal alignment of foot is necessary to evaluate the longitudinal arch angle and to prevent overuse injuries related to pes planus. The longitudinal arch of foot is supported not only by intrinsic muscles such as the abductor hallucis (AbdH), and extrinsic muscles such as the tibialis anterior/posterior but also by passive structure including the plantar fascia and spring ligament $[1,3,4]$. The longitudinal arch training has been recommended to prevent chronic pain in the foot and ankle [5], to improve balance in the standing position [6], and to prevent damage of the musculoskeletal system [7]. Previous studies have reported that the strength of the dorsiflexion and $\mathrm{AbdH}$ of individuals with collapsed longitudinal arch was weaker than those of normal individuals [5-7]. In particular, the weakness of the $\mathrm{AbdH}$ along with pes planus due to the collapse of the longitudinal arch may contribute to foot or ankle joint damage or sprain $[7,8]$.

The misaligned foot have the characteristic of collapsed longitudinal arch in the standing position. The $\mathrm{AbdH}$ is one of the muscles that plays important roles in not only maintaining the stability of the foot in a standing position but also in improving the alignment of the foot $[9,10]$. The $\mathrm{AbdH}$ contraction, which originates in the calcaneus bone and inserts into the great toe, contribute to flexion and abduction of the first metatarsophalangeal (MTP) joint [1], which moves the origin and insertion regions of $\mathrm{AbdH}$ close, and consequently increases and sup- 
ports the longitudinal arch [1,5]. Following surgery of the foot and ankle joint because of arthritis and sprain, factors such as weakness of the intrinsic muscles of the foot and functional limitations of the dorsiflexion affect the weakness of the $\mathrm{AbdH}$ [11]. In the previous studies, to strengthen the AbdH muscle, the short-foot exercise (SF), which pull the first metatarsal head toward the heel without toe flexion, and the toe spread out exercise (TSO), which perform abduction and flexion of the first and fifth metatarsophalangeal joints, are often investigated in the clinical rehabilitation [9].

However, the SF and TSO were considered without the fatigue of the $\mathrm{AbdH}$ muscle in individuals with pes planus, because in individuals with pes planus, the foot structure could not support the longitudinal arch in standing position [10]. The $\mathrm{AbdH}$ muscle may be in insufficient activation during these exercises. The fatigue of the $\mathrm{AbdH}$ [12], and decreased muscle activity of the AbdH [13], exacerbated the longitudinal arch in the standing position, which implies the important role of the $\mathrm{AbdH}$ in supporting the longitudinal arch. The overactivation of extrinsic muscles can be occurred to compensate for a weakened $\mathrm{AbdH}$ in individuals with pes planus $[14,15]$. The prolonged overactivation of extrinsic muscles including tibialis anterior and posterior results in muscle fatigue, consequently causing tendinitis, overuse injury $[11,16]$. Thus, AbdH strengthening exercise is important to support the longitudinal arch and prevent secondary injuries in individuals with pes planus [15]. Therefore, to induce maximal effort in the $\mathrm{AbdH}$, the arch support for the longitudinal arch of foot is required during TSO in standing position in individuals with pes planus.

In addition, sufficient understanding of the mechanism of such compensatory movements is essential to correctly evaluate and interpret the function of the $\mathrm{AbdH}[9,14]$. Based on the studies conducted to date, there has been no study that has investigated the effect of the longitudinal arch support on the strength and muscle activity of individuals with pes planus while performing TSO in the standing position. To comprehensively manage the $\mathrm{AbdH}$, it is essential to investigate the effect of the support of the longitudinal arch on the strength and muscle activity of the $\mathrm{AbdH}$ while performing TSO in the state of supporting weight [15]. The longitudinal arch of the foot contributes to the stability and balance of the foot in the standing position. Furthermore, together with the longitudinal arch, the AbdH provides dynamic stability to the ankle joint and metatarsal joint during functional movements, such as walking and running.

The purpose of this study is to investigate the effect of the support of the longitudinal arch using insole on the muscle activity of the AbdH while individuals with pes planus perform TSO in the standing position. The hypothesis of this study is that providing longitudinal arch support using insole to individuals with pes planus while performing the TSO in the standing position could improve the strength and muscle activity of the AbdH more than while it is not provided. The results of this study clinically suggest that the management of the $\mathrm{AbdH}$ is more effective while individuals with pes planus perform the TSO with the longitudinal arch using insole formed in the standing position.

\section{MATERIALS AND METHODS}

\section{Subjects}

For determining the number of the participants, power was set at 0.80 , alpha level at 0.05 , and effect size at 1.42 , which are required elements of the G*Power program (ver. 3.1.2, Franz Faul; University of Kiel, Kiel, Germany). We found that a minimum of 15 participants were required for the experiment; considering the possibility of dropout, a total of 20 participants with pes planus (age: $25.1 \pm 5.2$ years, weight: $67.3 \pm 8.6$ $\mathrm{kg}$, height: $170.1 \pm 6.9 \mathrm{~cm}$, males: 10 , females: 10) were selected. The criterion of pes planus has a calcaneal stance angle during resting position with at least $4^{\circ}$ and a navicular drop (ND) with at least $13 \mathrm{~mm}$.

The inclusion criteria were as follows: (1) No limited range of motion of the foot and ankle joint, (2) no previous history of ankle joint surgery. The exclusion criteria were as follows: (1) history of musculoskeletal fracture, (2) pain in any part of the body experienced during the execution of the experiment.

The process of the experiment was explained in detail to the participants. All participants provided written consent according to the tenets of the Declaration of Helsinki. This study was approved by the Institutional Review Board (approval no. 1041231-200218-HR-103-01).

\section{Instruments}

\section{1) Identification of the location of the AbdH using ultrasound}

To confirm the precise muscle belly of the individual muscle, a 7.5-MHz linear head connected to the ultrasound (Q30; SG 
Healthcare Co., Ltd., Seoul, Korea) was used. After marking the precise area identified via ultrasound with a pen, the ultrasound gel was removed for attaching the electromyogram (EMG) patch.

\section{2) Measurement of the muscle activity of the $\mathrm{AbdH}$}

Muscle activity was measured using EMG-feedback tools (wireless EMG system 100RT; BTS, Millan, Italy). A software was used to analyze the muscle activity of the AbdH. Filter $(60 \mathrm{~Hz})$ and band pass filter type extraction rate $(1,024 \mathrm{~Hz})$ were set and the collected muscle activity was treated with root-meansquare. To minimize the resistance at the location where the EMG patch was attached, the location was shaved and disinfected with alcohol cotton. Two electrodes were attached in parallel with the direction of the muscular fiber. The maximal voluntary isometric contraction (\%MVIC) method was used to normalize the data. When the hallux was at its maximum level of abduction of the great toe from the axial line of the foot, a resisting force was exerted by the investigator's hand to the center of its the medial side of the first metatarsal and proximal phalanx in sitting position [14]. The mean obtained after a total of three measurements was used to determine \%MVIC (Figure 1).

\section{3) Measurement of the strength of the $\mathrm{AbdH}$}

The strength of the maximum isometric contraction of the $\mathrm{AbdH}$ was measured using the Smart KEMA Pulling Sensor (Factorial Holdings Co., Ltd., Seoul, Korea) (Figure 2). A thin strap connected to the pulling sensor was placed between the metatarsophalangeal joint and the interphalangeal joint of the hallux, i.e., at the center of its proximal phalanx. For the measurement of the selective strength of the $\mathrm{AbdH}$, the first metatarsal bone was fixed using a non-elastic fixation belt to prevent adduction of ankle joint. To minimize the errors in measurement, the area of the toe joints in contact with the strap was marked. The length of the strap was controlled in the neutral position of the hallux in the standing position. The maximum weight of measurement determined using the Smart KEMA Pulling Sensor was $100 \mathrm{~kg}$, with disappearance and precision of less than $0.1 \mathrm{~kg}$. To provide the support for the longitudinal arch of foot, the insole formed with the same height as the height of the navicular bone measured in the sitting position was used [13].

\section{Procedures}

As all participants had both feet with pes planus, the maximum isometric muscle contraction of the $\mathrm{AbdH}$ of the dominants was measured. The order for applying the longitudinal

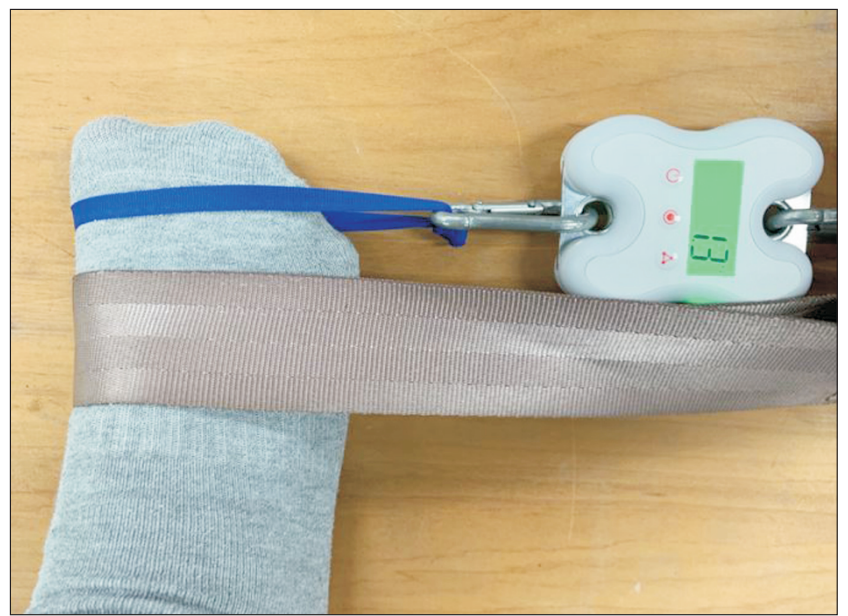

Figure 2. The strength measurement of abductor hallucis.
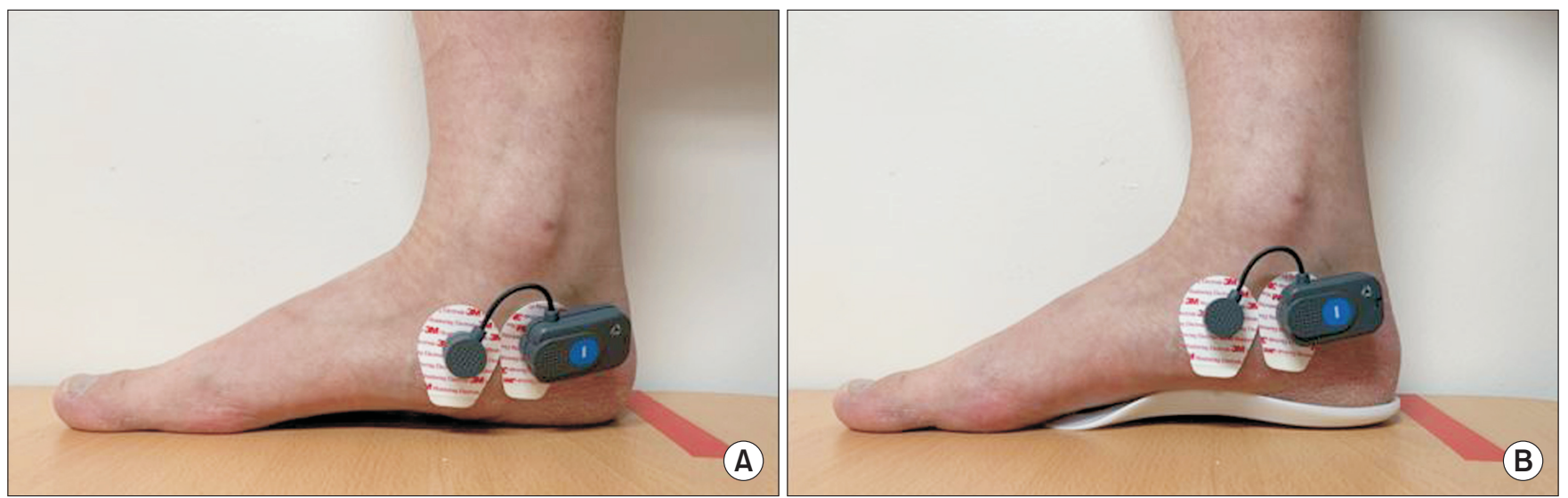

Figure 1. The muscle activity of the AbdH muscle in individuals with pes planus (A) without arch support, (B) with arch support by using the insole. 
arch support was determined via randomization. Each participant practiced TSO while the longitudinal arch support using insole was provided. The insole for longitudinal arch support was used according to the height of the navicular bone in the sitting position. Then, the participant rehearsed for the experiment in the exact same manner to precisely execute the procedure. The participant had weight support by standing comfortably on both feet for measurement. Then, the strength and muscle activity of the $\mathrm{AbdH}$ in the dominant foot was measured via maximum isometric muscle contraction. The participant performed the TSO with the support for the longitudinal arch. The spreading of the TSO was maintained for 5 seconds. The measurement of the muscle activity of the $\mathrm{AbdH}$ was executed together with the measurement of the strength. The strength and muscle activity were simultaneously measured using a tensiometer and EMG devices with three times, and the data was averaged. After one-time execution, a 10-minute resting period was provided.

\section{Statistical Analysis}

The SPSS software (ver. 18.0; IBM Co., Armonk, NY, USA) was used for statistical analysis. The normal distribution of the data measured was confirmed via the one-sample Kolmogorov-Smirnov test. The paired t-test was used to confirm whether there are statistically significant differences between the conditions (longitudinal arch support vs. no support). The significance level of the statistics was set at $\mathrm{p}<0.05$.

\section{RESULTS}

There was a statistically significance increase in the strength and muscle activity of the $\mathrm{AbdH}$ while the longitudinal arch support was provided than that while the longitudinal arch support was not provided (Tables 1, 2) $(\mathrm{p}<0.05)$.

Table 1. The muscle activity of the abductor hallucis in individuals with pes planus

\begin{tabular}{ccccc}
\hline $\begin{array}{c}\text { The muscle activity } \\
\text { (\%MVIC) }\end{array}$ & $\begin{array}{c}\text { Without arch } \\
\text { support }\end{array}$ & $\begin{array}{c}\text { With arch } \\
\text { support }\end{array}$ & t value & $p$-value \\
\hline Abductor hallucis & $28.32 \pm 4.13$ & $41.03 \pm 8.51$ & -7.42 & $<0.05$ \\
\hline
\end{tabular}

Values are presented as mean \pm standard deviation. MVIC, maximal voluntary isometric contraction.

\section{DISCUSSION}

Adequate strength of the $\mathrm{AbdH}$ is an important factor for rehabilitation of the pes planus. However, clinical evaluation of the strength and muscle activity of the individuals with pes planus required consideration of the longitudinal arch. This study aimed to determine the effect of the longitudinal arch support using insole on the strength and muscle activity of the $\mathrm{AbdH}$ in individuals with pes planus in the standing position. While individuals with pes planus performed TSO, the strength and muscle activity of the $\mathrm{AbdH}$ showed a statistically significant increase with the longitudinal arch support (strength increased by $1.43 \mathrm{~kg}$ and muscle activity increased by 12.71\%) (p $<0.05)$. As a result, the muscle activity of the $\mathrm{AbdH}$ increased at the alignment, allowing its ideal contraction. This could be considered to have positively influenced the increased strength of the $\mathrm{AbdH}[17]$.

There are several reasons for the difference in the strength and muscle activity of the $\mathrm{AbdH}$ depending on the longitudinal arch support during the performance of TSO. First, it could be explained with regards to the length-tension relationship. The $\mathrm{AbdH}$ plays an important stabilizing role in the first metatarsal joint, particularly in the one leg standing position [13]. The $\mathrm{AbdH}$ contraction, which originates in the calcaneus bone and inserts into the great toe, contribute to flexion and abduction of the first MTP joint [1], which moves the origin and insertion regions of $\mathrm{AbdH}$ close, and consequently increases and supports the longitudinal arch [1,5]. While the longitudinal arch support was not provided, pronation of ankle joint and the pes planus in the standing position were occurred because of falling of the navicular bone. This became a lengthened position of the $\mathrm{AbdH}$. However, while the longitudinal arch of the foot is formed in the standing position during TSO, the pronation of the ankle bone could be prevented via the longitudinal arch support against the weight $[13,18]$. As a result, the ideal alignment for effective contraction of the $\mathrm{AbdH}$ in standing position was formed. The ideal alignment of the foot, which allows ef-

Table 2. The strength of the abductor hallucis in individuals with pes planus

\begin{tabular}{ccccc}
\hline $\begin{array}{c}\text { The strength } \\
(\mathrm{kg})\end{array}$ & $\begin{array}{c}\text { Without arch } \\
\text { support }\end{array}$ & $\begin{array}{c}\text { With arch } \\
\text { support }\end{array}$ & t value & $\mathrm{p}$-value \\
\hline Abductor hallucis & $1.36 \pm 0.84$ & $2.79 \pm 0.34$ & -6.13 & $<0.05$ \\
\hline Values are presented as mean \pm standard deviation.
\end{tabular}


fective contraction of the $\mathrm{AbdH}$, may be considered to be an important factor for increasing the muscle activity of the $\mathrm{AbdH}$. The improvement of the AbdH muscle activity may be considered to have affected the increase in the strength of the AbdH.

Second, the reason could be explained with regards to biomechanical factors. The stability of the foot contributed to the activation of its intrinsic muscles during TSO. In particular, as the $\mathrm{AbdH}$ is attached to the center of the metatarsal bone, it is affected more from the activation of the intrinsic muscle $[19,20]$. In other previous studies, the muscle activity of the AbdH was greater with TSO compared to SF [21]. While individuals with pes planus performed the TSO, longitudinal arch using insole was formed, thereby activating the intrinsic muscle, which also affected the improvement of the muscle activity of the AbdH. The longitudinal arch support using insole provided an effective alignment that allows the co-contraction of the intrinsic muscle while performing the TSO in standing position $[20,21]$. As the results of this study, the longitudinal arch of foot may be considered to affect the increase in the strength and muscle activity of the $\mathrm{AbdH}[18,22]$.

Based on previous studies, when performing the SF for the activation of the abductor hallucis, the longitudinal arch was increased more in the plantar flexion than in the neutral position of the ankle, and its contribution to the increased muscle activity was confirmed [23]. In addition, based on recent study by Kang et al. [24], when the healthy group and the group with hallux valgus performed the TSO in three positions (plantar flexion, neutral, and dorsiflexion), a significantly higher increase in the cross-sectional area of the $\mathrm{AbdH}$ measured via ultrasound was confirmed in plantar flexion than in the other positions (neutral and dorsiflexion) in both groups. The increase in the ultrasonic cross-sectional area of the AbdH may be considered to have a very high correlation with muscle activity [24]. Although it was difficult to directly compare this result with those of previous studies, performing SF and TSO in plantar flexion to form the longitudinal arch is consistent with this study. This study also confirmed the increased strength and muscle activity of the $\mathrm{AbdH}$ via the longitudinal arch formed by using insole while individuals with pes planus performed the TSO in the standing position.

This study has several limitations. First, only individuals with pes planus were selected for the experiment. For further studies, it is necessary to investigate the effects of longitudinal arch on the strength and muscle activity of the $\mathrm{AbdH}$ with other impairments such as hallux valgus and hammer toe. Second, the insole for longitudinal arch support was used according to the height of the navicular bone in the sitting position. Further studies should investigate the correlation depending on the height of the support for the longitudinal arch. Third, the individuals were relatively young (in their 20-30s). Therefore, there is a limitation with regards to applying the results to the elderly population. Therefore, further studies should compare the results according to different age groups.

\section{CONCLUSIONS}

Longitudinal arch support using insole for individuals with pes planus improved the muscle activity and muscle strength of the $\mathrm{AbdH}$ while performing the TSO in the standing position, the TSO with longitudinal arch support using insole may be highly recommended in individuals with pes planus.

\section{ACKNOWLEDGEMENTS}

This research was supported by the Academic Research fund of Hoseo University in 2019 (2019-0807).

\section{CONFLICTS OF INTEREST}

No potential conflict of interest relevant to this article was reported.

\section{AUTHOR CONTRIBUTIONS}

Conceptualization: IJ. Data curation: IJ. Formal analysis: IJ. Funding acquisition: KK. Investigation: IJ. Methodology: KK. Project administration: IJ. Supervision: KK. Validation: KK. Writing - original draft: IJ. Writing - review \& editing: KK.

\section{ORCID}

In-cheol Jeon, https://orcid.org/0000-0002-6549-6866

\section{REFERENCES}

1. Neumann DA. Kinesiology of the musculoskeletal system: foundations for rehabilitation. 2nd ed. St. Louis: Mosby; 2010.

2. Barton CJ, Bonanno D, Levinger P, Menz HB. Foot and ankle 
characteristics in patellofemoral pain syndrome: a case control and reliability study. J Orthop Sports Phys Ther 2010;40(5):286-96.

3. Gray EG, Basmajian JV. Electromyography and cinematography of leg and foot ("normal" and flat) during walking. Anat Rec 1968;161(1):1-15.

4. Murley GS, Landorf KB, Menz HB, Bird AR. Effect of foot posture, foot orthoses and footwear on lower limb muscle activity during walking and running: a systematic review. Gait Posture 2009;29(2):172-87.

5. Sahrmann SA. Diagnosis and treatment of movement impairment syndromes. St. Louis: Mosby; 2001.

6. Ekstrom RA, Donatelli RA, Carp KC. Electromyographic analysis of core trunk, hip, and thigh muscles during 9 rehabilitation exercises. J Orthop Sports Phys Ther 2007;37(12):75462.

7. Leetun DT, Ireland ML, Willson JD, Ballantyne BT, Davis IM. Core stability measures as risk factors for lower extremity injury in athletes. Med Sci Sports Exerc 2004;36(6):926-34.

8. Kisner C, Colby LA. Therapeutic exercise: foundations and techniques. 6th ed. Philadelphia: F.A. Davis; 2012.

9. Jung DY, Kim MH, Koh EK, Kwon OY, Cynn HS, Lee WH. A comparison in the muscle activity of the abductor hallucis and the medial longitudinal arch angle during toe curl and short foot exercises. Phys Ther Sport 2011;12(1):30-5.

10. Coughlin MJ, Saltzman CL, Nunley JA 2nd. Angular measurements in the evaluation of hallux valgus deformities: a report of the ad hoc committee of the American Orthopaedic Foot \& Ankle Society on angular measurements. Foot Ankle Int 2002;23(1):68-74.

11. Mann RA, Coughlin MJ. Adult hallux valgus. In: Coughlin MJ, Mann RA, editors. Surgery of the foot and ankle. 7th ed. St. Louis: Mosby; 1999;100-28.

12. Headlee DL, Leonard JL, Hart JM, Ingersoll CD, Hertel J. Fatigue of the plantar intrinsic foot muscles increases navicular drop. J Electromyogr Kinesiol 2008;18(3):420-5.

13. Sahrmann S. Movement system impairment syndromes of the extremities, cervical and thoracic spines. London: Elsevier Health Sciences; 2010.

14. Kendall FP, McCreary EK, Provance PG, Rodgers MM, Romani WA. Muscles: testing and function with posture and pain. 5th ed. Baltimore: Lippincott Williams \& Wilkins; 2005.

15. Tehraninasr A, Saeedi H, Forogh B, Bahramizadeh M, Keyhani MR. Effects of insole with toe-separator and night splint on patients with painful hallux valgus: a comparative study. Prosthet Orthot Int 2008;32(1):79-83.

16. Kim MH, Kwon OY, Kim SH, Jung DY. Comparison of muscle activities of abductor hallucis and adductor hallucis between the short foot and toe-spread-out exercises in subjects with mild hallux valgus. J Back Musculoskelet Rehabil 2013;26(2):163-8.

17. Lee JE, Park GH, Lee YS, Kim MK. A comparison of muscle activities in the lower extremity between flat and normal feet during one-leg standing. J Phys Ther Sci 2013;25(9):105961.

18. Heo HJ, Koo YM, Yoo WG. Comparison of selective activation of the abductor hallucis during various exercises. J Phys Ther Sci 2011;23(6):915-8.

19. Jeon I, Jang J. Comparison of tibialis anterior muscle activity and dorsiflexor strength according to toe postures. J Musculoskelet Sci Technol 2020;4(1):13-7.

20. Lee JH, Cynn HS, Yoon TL, Choi SA, Kang TW. Differences in the angle of the medial longitudinal arch and muscle activity of the abductor hallucis and tibialis anterior during sitting short-foot exercises between subjects with pes planus and subjects with neutral foot. J Back Musculoskelet Rehabil 2016;29(4):809-15

21. Cha S, Kang M, Moon D, Oh J. Effect of the short foot exercise using an electromyography biofeedback on medial longitudinal arch during static standing position. Phys Ther Korea 2017;24(1):9-18.

22. Arinci Incel N, Genç H, Erdem HR, Yorgancioglu ZR. Muscle imbalance in hallux valgus: an electromyographic study. Am J Phys Med Rehabil 2003;82(5):345-9.

23. Yoon H, Kim J, Park J, Jeon H. Comparison of the foot muscle EMG and medial longitudinal arch angle during short foot exercises at different ankle position. Phys Ther Korea 2017;24(4):46-53.

24. Kang ST, Kang SY, Kim KS, Jeon IC, Choung SD. Changes in the cross-sectional area of the abductor hallucis during the toespread-out exercise at different ankle positions. J Korean Soc Phys Med 2020;15(2):121-8. 